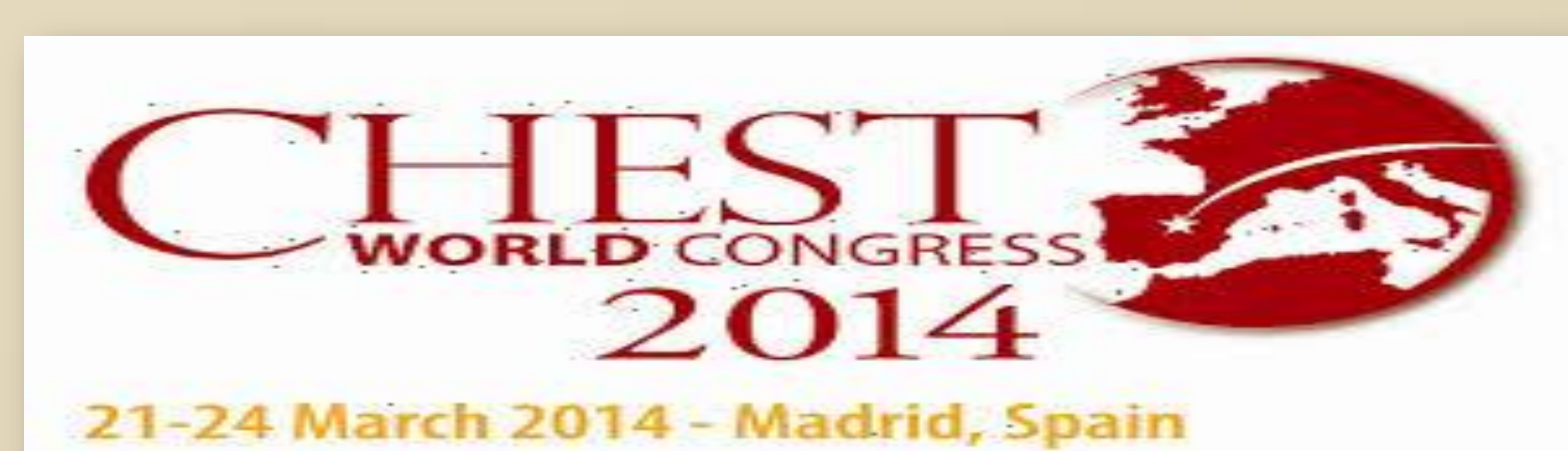

\title{
Non Invasive Ventilation during exercise in COPD patients: A Systematic review with Meta-analysis
}

\section{Sara Correia ${ }^{1}, \mathbf{M}^{\mathrm{a}}$ Teresa Tomás² , Elizabete Carolino3}

\author{
'ResMed - Porto, Portugal; PT, MsC : (sara_correia@live.com.pt) \\ 2 Lisbon Higher School of Health Technology (ESTeSL - IPL) - Scientific Area of Physiotherapy; PT, PhD (teresa.tomas@estesl.ipl.pt) \\ 3 - Lisbon Higher School of Health Technology (ESTeSL - IPL) - Scientific Area of Mathematics, PhD (etcarolino@estesl.ipl.pt)
}

\section{Purpose}

COPD is a major cause of morbidity and mortality worldwide, representing a major public health problem due to the high health and economic resource consumption. Pulmonary rehabilitation is a standard care recommendation for these patients, in order to control the symptoms and optimize the functional capacity, reducing healthcare costs associated with exacerbations and activity limitations and participation. However, in patients with severe COPD exercise performance can be difficult, due to extreme dyspnea, decreased muscle strength and fatigue. In addition, hypoxemia and dyspnea during efforts and daily activities may occur, limiting their quality of life. Thus, NIV have been used as adjunct to exercise, in order to improve exercise capacity in these patients. However, there is no consensus for this technique recommendation $(1,2)$

Our objective was to verify whether the use of NIV during exercise is effective than exercise without NIV in dyspnea, walked distance, blood gases and health status in COPD patients, through a systematic review and meta-analysis.
\end{abstract}

\section{Methods}

A systematic search for RCT published between 2002 and 2012 was performed in MEDLINE, PEDro, Cochrane, Science Direct and B-On databases, with "NIV", "Exercise", "Exercise training" and "COPD" as keywords. Only studies associating NIV and exercise in COPD population, accessing 6MWT, SGRQ, ABG or SpO2 were included. We used descriptive statistics and the "comprehensive meta analysis version 2.0" software for meta-analysis.

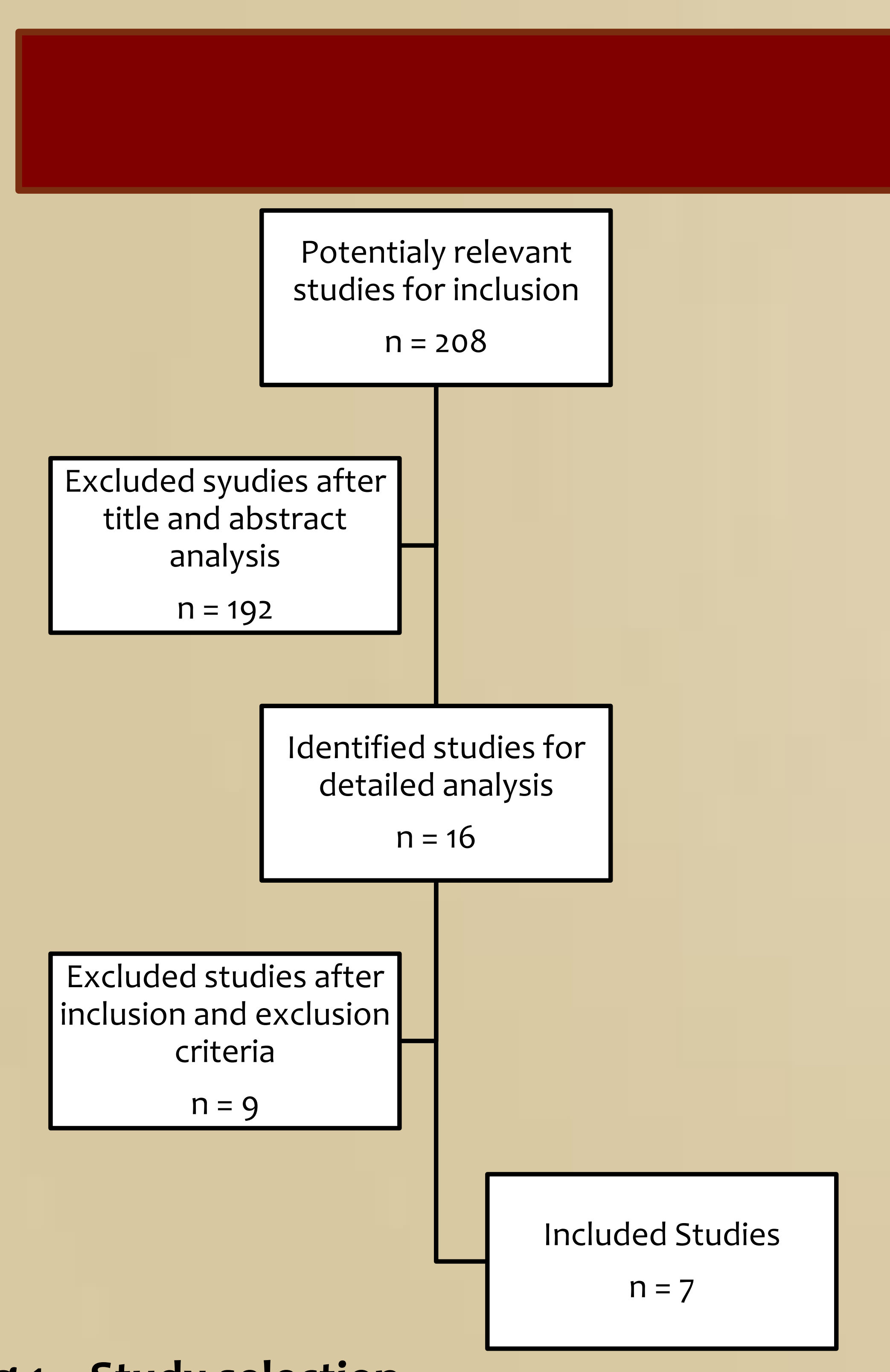

Fig.1 - Study selection.

From 208 identified randomized controlled trials, only 7 were included. Of these, only 4 allowed the meta-analysis for $\mathrm{PaCO} 2$ and dyspnea. 6 of the 7 studies indicated the benefit of NIV compared to the control group. From these, 2 studies evaluated the acute response to exercise and other 4 the chronic response.

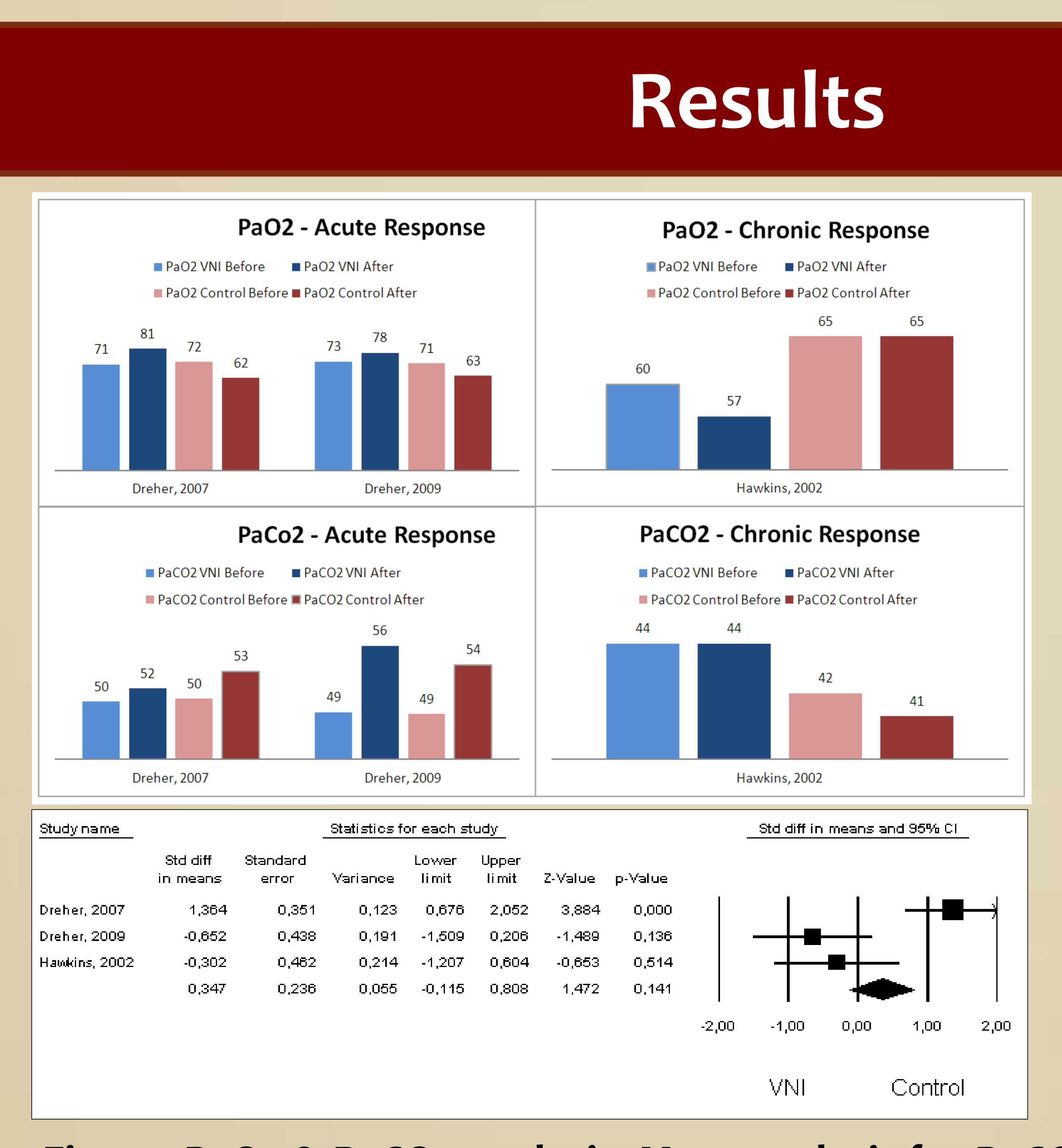

Fig. 2 - PaO2 \& PaCO2 analysis. Meta-analysis for PaCO2.

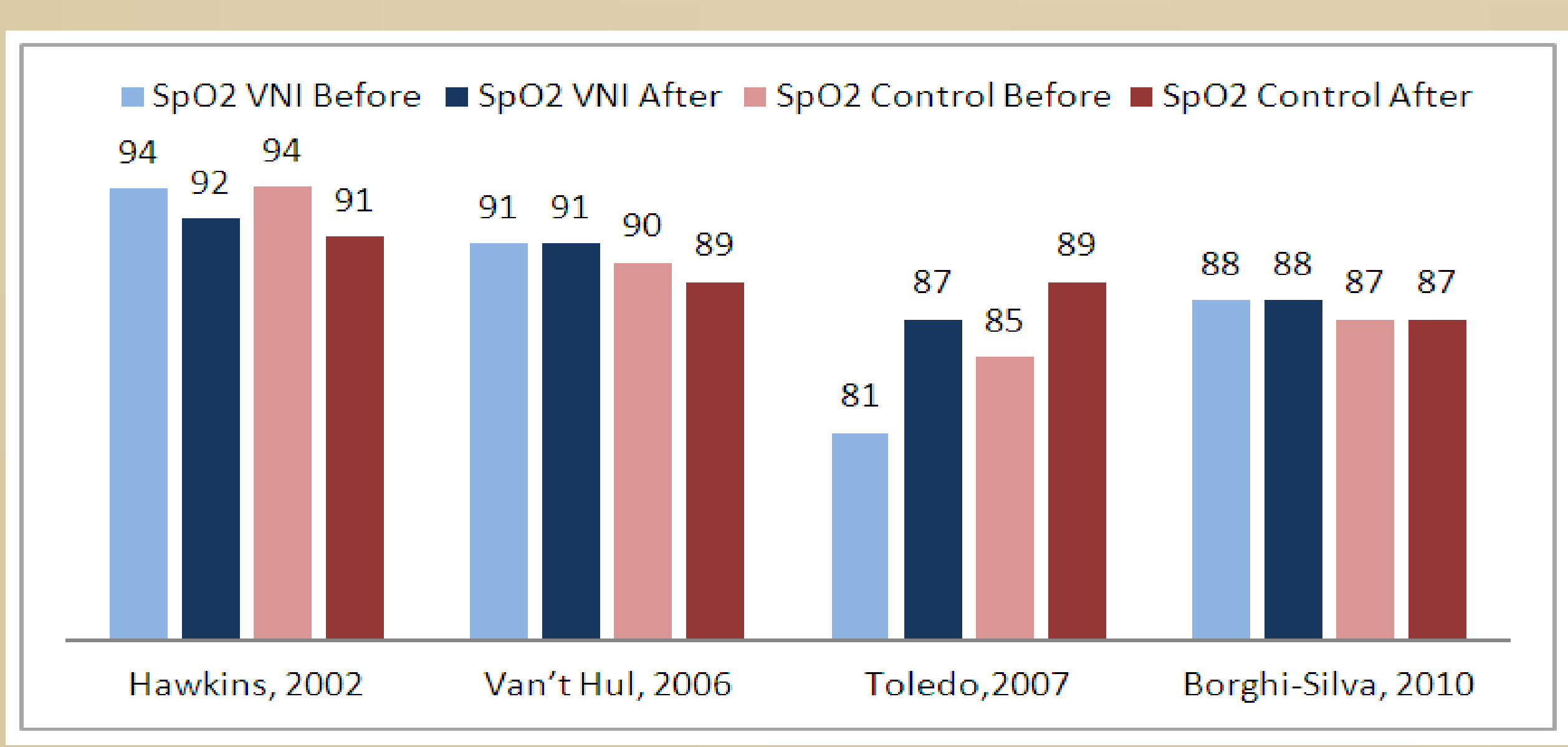

Fig. 3 - SpO2 analysis.

NIV prevents Hipoxia and maintain $\mathrm{SpO}_{2}$ with or without aditional $\mathrm{O}_{2}$

No estatistical diferences were found for $\mathrm{PaCO}_{2}$

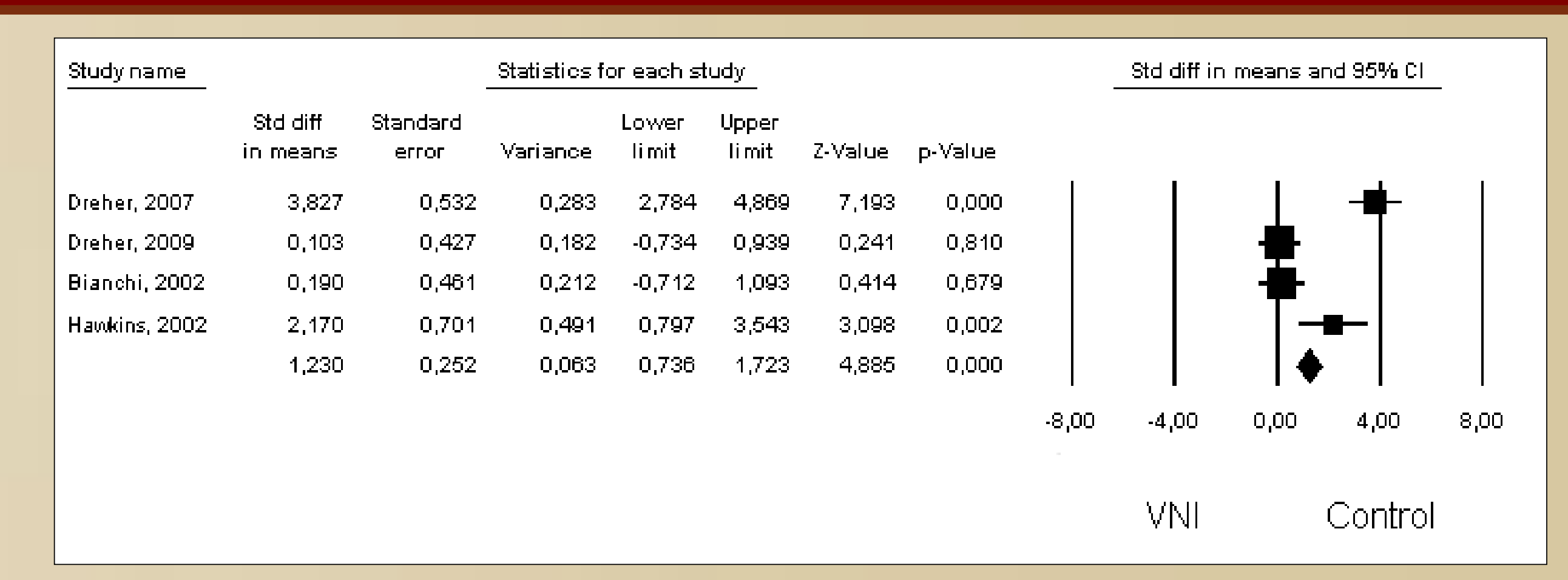

Fig. 4 -Meta-analysis for Dyspnea.

We found a higher increase in dyspnea in the control group

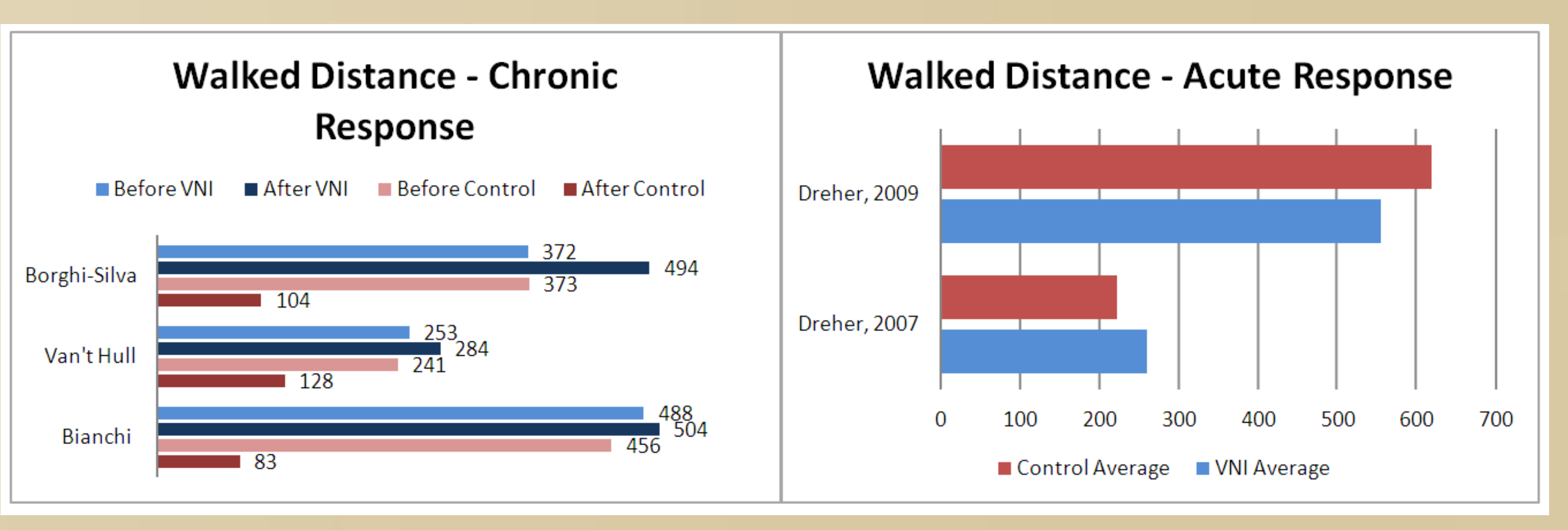

Fig. 5 -Walked distance.

Patients under NIV walked longer than control group

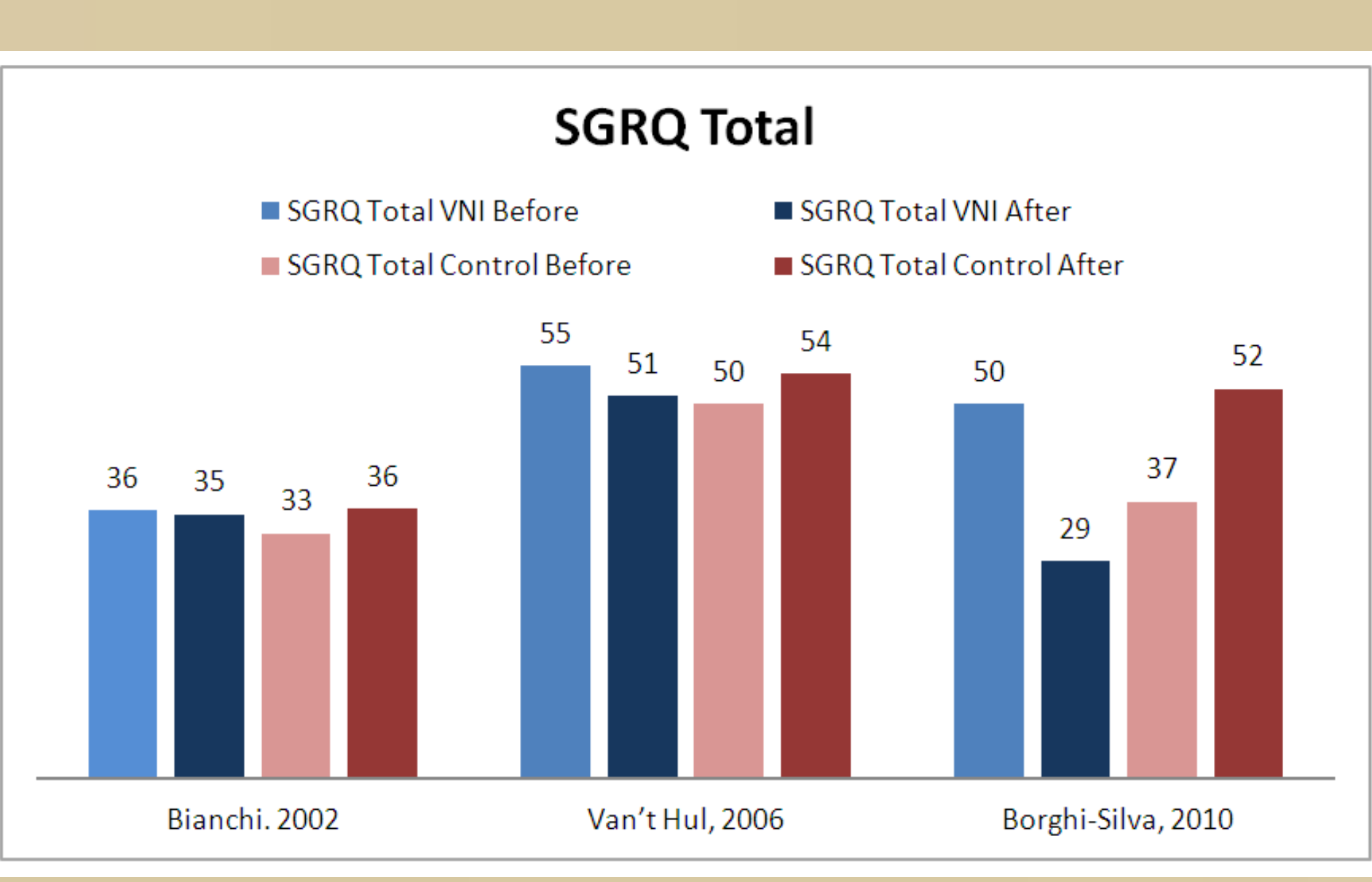

Fig. 6 -Quality of Life

\section{Conclusions and Clinical Implications}

-We verified a positive influence of NIV during exercise, both in terms of acute and chronic responses to exercise in patients either hypercapnic and normocapnic, regardless of conducting training programs of exercise.

CLINICAL IMPLICATIONS: The usage of NIV during exercise in COPD patients seems to be beneficial.

ACKNOWLEGMENT: We would like to thank Dr. Michael Dreher, Dr. Luca Bianchi and specially to Dr. Peter Hawkins for their kindness and availability sending the relevant information to perform this study. 